EESTI NSV TEADUSTE AKADEEMIA TOIMETISED. IX KOIDE

FOOSIKALIS-MATEMAATILISTE JA TEHNILISTE TEADUSTE SEERIA, 1960, NR. 3

ИЗВЕСТИЯ АКАДЕМИИ НАУК ЭСТОНСКОЙ ССР. ТОМ IХ

СЕРНЯ ФИЗНКО-МАТЕМАТИЧЕСКИХ И ТЕХНИЧЕСКИХ НАУК, 1960, № 3

\title{
К ТЕОРИИ ОТРАЖЕНИЯ СВЕТА ТОНКИМ МЕТАЛЛИЧЕСКИМ СЛОЕМ
}

\author{
(Ответ на статью П. Г. Карда)
}

\author{
АНТОНИН ВАШИЧЕК, \\ доктор физико-математических наук
}

В 1958 году в этом журнале была опубликована статья П. Г. Карда [1] с возражениями против ревизии формул Мурмана для отражения света тонким металлическим слоем, предпринятой автором настоящей статьи. Возражения, выдвинутые Кардом против ревизии упомянутых формул Мурмана, были особенно серьезными в заключительной части его работы: по Карду, ревизия касается самых основ общепринятой теории Максвелла. Эти возражения столь серьезны, что вынуждают автора высказать свое мнение о статье Карда, с тем чтобы показать, что ревизия не касается основ теории Максвелла.

\section{Введение}

Автор впервые выступил против формул Мурмана для отражения света тонким металлическим слоем уже более десяти лет назад, в то время, когда эта отрасль оптики только начинала разрабатываться. Нами было обнаружено, что фазы в формулах Мурмана неверны, что и было доказано с помощью энергетической проверки. Вскоре после этого против ревизии формул Мурмана выступили Врьба $\left[{ }^{2}\right]$ и Книттл [4] и позже Кард [']. Развитие знаний по оптике тонких слоев за последние десять лет показало, что наши возражения против теории Мурмана для отражения света тонким металлическим слоем вполне обоснованы. Особенно ярко свидетельствуют об этом численные расчеты зависимости отражательной и пропускательной способностей от толщины слоя, проделанные автором и его сотрудником Дунайским для нескольких металпов. Энергетическая проверка, подвергнутая критике Кардом, играет в настоящее время в нашем споре самую незначительную роль. Ряд проблем, связанных с этой проверкой, обсужден в нашей переписке с Кардом. Основные результаты своих работ автор настоящей статьи приводит в английском издании своей книги «Оптика тонких слоев» [5], где эти проблемы рассматриваются более подробно.

В настоящей статье выводы, приведенные в названной книге, дополняются еще с одной стороны: в ней рассматривается, как выглядят эти проблемы с точки зрения принципов обратимости Власова $\left[{ }^{7}\right]$ и Стокса [8]. Вполне очевидно, что непонимание различий между обоими принципами или же подмена их одного другим, как это делает в одной из своих последних работ Книттл [9], приводит к неверным результатам.

Известно, что для отражения света тонким слоем используется общая формула

$$
\mathrm{r}_{R}=r_{R} e^{i \bar{t}} R=\frac{\mathrm{r}_{R}^{\prime}+\mathrm{r}_{R}^{\prime \prime}\left(\mathrm{t}_{R}^{\prime} \mathrm{t}_{L}^{\prime}-\mathrm{r}_{R}^{\prime} \mathrm{r}_{L}^{\prime}\right) e^{-i x}}{1-\mathrm{r}_{L}^{\prime} \mathrm{r}_{R}^{\prime \prime} e^{-i x}}
$$

в которой индексы $R$ и $L$ относятся к комплексным амплитудам света, рассчитанным для поступления света справа (индекс $R$ ) или слева (индекс $L$ ). В формуле (1) не важно, относятся ли эти амплитуды к случаю простого (френелевского) раздела или к случаю гипотетического раздела, заменяющего систему тонких слоев диэлектрика. 


\section{1. Принципы обратимости}

В оптике тонких слоев мы встречаемся практически с двумя принципами обратимости, названными нами принципами Власова и Стокса. В случае принципа обратимости Власова для обратного хода световых лучей при переходе через границу раздела берем одинаковые комплексные амплитуды. В случае же принципа обратимости Стокса для обратного хода световых лучей через границу раздела необходимо взять комплексно сопряженные амплитуды. Это означает, что в случае принципа обратимости Стокса поляризация при обратном ходе света не меняется (положительная останется положительной, отрицательная отрицательной), в то время как в принципе обратимости Власова с изменением хода света она меняется (положительная становится отрицательной и наоборот). Различия обоих принципов обратимости наглядно показаны на фиг. 1 и 2.

Принцип обратимости Власова применяют обычно для простой френелевской границы раздела, когда соответствующие комплексные амплитуды отраженного и преломленного света в обоих направлениях $R$ и $L$ можно выразить непосредственно формулами Френеля. Например, для
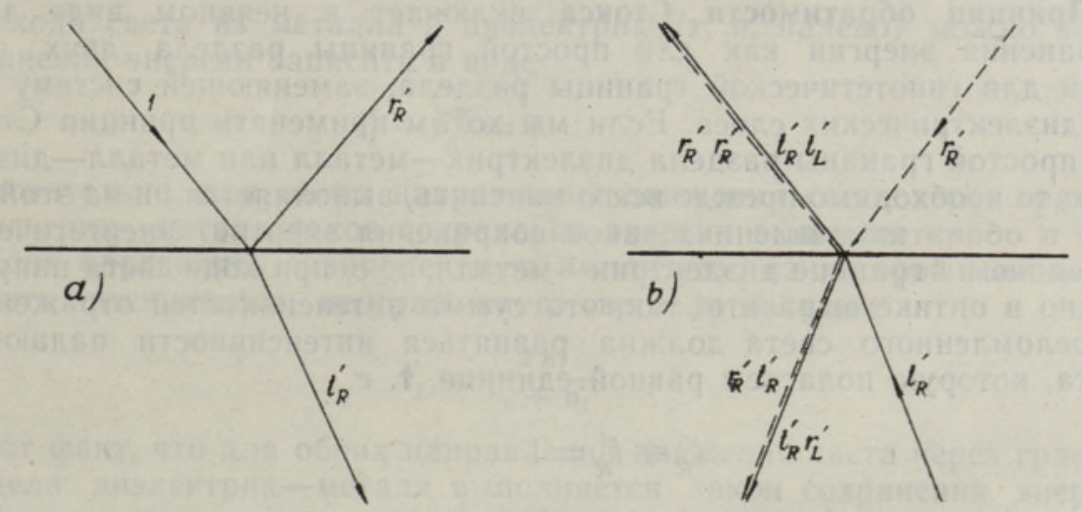

Фиг. 1. К принципу обратимости Власова.

нормального падения света из диэлектрика с показателем преломления $n_{0}$ на металл с комплексным показателем преломления $\mathrm{n}_{1}=n_{1}-i n_{1} k_{1}$ можно упомянутые соотношения Френеля для комплексных амплитуд отраженного света записать в виде

$$
\mathbf{r}_{R}^{\prime}=\frac{\mathrm{n}_{1}-n_{0}}{\mathrm{n}_{1}+n_{0}}=-\mathbf{r}_{L}^{\prime}
$$

и для комплексных амплитуд преломленного света получаем

$$
\mathbf{t}_{R}^{\prime}=\frac{2 n_{0}}{\mathbf{n}_{1}+n_{0}}, \quad \mathbf{t}_{L}^{\prime}=\frac{2 \mathbf{n}_{1}}{n_{0}+\mathbf{n}_{1}}
$$

Дунайский $\left[{ }^{10}\right]$ приводит следуюшие соотношения для напряженностей электрического и магнитного полей [ $\left.{ }^{7}\right]$ при обратном ходе луча:

$$
\mathrm{E} \rightarrow \mathrm{E}, \mathrm{H} \rightarrow-\mathrm{H} \text { или } \mathrm{E} \rightarrow-\mathrm{E}, \mathrm{H} \rightarrow \mathbf{H} .
$$

В обоих случаях согласно фиг. 1 получаем из принципа обратимости 
Власова соотношения

$$
\begin{gathered}
\mathbf{t}_{R}^{\prime} \mathbf{t}_{L}^{\prime}+\mathbf{r}_{R}^{\prime 2}=1 \\
\mathbf{r}_{L}^{\prime}=-\mathbf{r}_{R}^{\prime}
\end{gathered}
$$

Эти соотношения содержат комплексные амплитуды $\mathbf{r}_{L}^{\prime}$ и $\mathbf{t}_{L}^{\prime}$ как неизвестные. Для френелевской границы раздела эти соотношения, как правило, не применяются, так как комплексные амплитуды $\mathbf{r}_{L}^{\prime}$ и $\mathbf{t}_{L}^{\prime}$, как это видно из формул (2) и (3), можно вычислить непосредственно.

Если подставить эти соотношения в общую формулу для отражения света тонким металлическим слоем, то получим общеизвестную формулу Мурмана

$$
\mathbf{r}=r e^{i \bar{\delta}}=\frac{\mathbf{r}^{\prime}+\mathbf{r}^{\prime \prime} e^{-i \mathbf{x}}}{1+\mathbf{r}^{\prime} \mathbf{r}^{\prime \prime} e^{-i \mathbf{x}}}
$$

(если опустить индекс $R$, одинаковый для всех амплитуд). Сдвиг фазы световых лучей тонким металлическим слоем является комплексным числом х. Формула (6) верна и для тонкого слоя диэлектрика. В этом случае амплитуды $r^{\prime}, r^{\prime \prime}$ и сдвиг фазы $x$ суть вещественные числа.

Принцип обратимости Стокса включает в неявном виде закон сохранения энергии как для простой границы раздела двух сред, так и для гипотетической гранишы раздела, заменяющей систему тонких диэлектрических слоев. Если мы хотим применять принцип Стокса для простой границы раздела диэлектрик-металл или металл-диэлектрик, то необходимо прежде всего выяснить, выполняется ли на этой границе в обоих направлениях закон сохранения энергии. Энергетическое состояние на границе диэлектрик-металл, т. е. при ходе света направо, можно в оптике выразить так, что сумма интенсивностей отраженного и преломленного света должна равняться интенсивности падающего света, которую полагаем равной единице, т. е.

$$
I_{R}^{r}+I_{R}^{t}=1
$$

Интенсивность отраженного света дана формулой

$$
I_{R}^{r}=\frac{\left(n_{1}-n_{0}\right)^{2}+n_{1}^{2} k_{1}^{2}}{\left(n_{1}+n_{0}\right)^{2}+n_{1}^{2} k_{1}^{2}}
$$

и интенсивность преломленного света -

$$
I_{R}^{t}=\frac{4 n_{0} n_{1}}{\left(n_{1}+n_{0}\right)^{2}+n_{1}^{2} k_{1}^{2}}
$$

Нетрудно убедиться в том, что закон сохранения энергии, выраженный формулой (7), действительно выполняется.

Если рассматривать направление движения света налево (т. е. в обратную сторону - из металла в диэлектрик), то условия усложняются, так как нужно учитывать еще интерференционную интенсивность $I_{L}^{\text {int }}$, так что закон сохранения энергии можно записать в более общем виде [ $\left.{ }^{9}\right]$

$$
I_{L}^{r}+I_{L}^{t}+I_{L}^{\mathrm{int}}=1
$$


Отдельные интенсивности определяются следующими формулами: интенсивность отраженного света

$$
I_{L}^{r}=\frac{\left(n_{0}-n_{1}\right)^{2}+n_{1}^{2} k_{1}^{2}}{\left(n_{0}+n_{1}\right)^{2}+n_{1}^{2} k_{1}^{2}}
$$

интенсивность преломленного света

$$
I_{L}^{t}=\frac{4\left(n_{1}^{2}+n_{1}^{2} k_{1}^{2}\right)}{\left(n_{0}+n_{1}\right)^{2}+n_{1}^{2} k_{1}^{2}} \frac{n_{0}}{n_{1}}
$$

и интерференционная интенсивность

$$
I_{L}^{\mathrm{int}}=-\frac{4 n_{1}^{2} k_{1}^{2}}{\left(n_{0}+n_{1}\right)^{2}+n_{1}^{2} k_{1}^{2}} \frac{n_{0}}{n_{1}}
$$

Если соединить, как это делает Дунайский $\left[{ }^{10}\right]$, интерференционную интенсивность $I_{L}^{\text {int }}$ и интенсивность преломленного света $I_{L}^{t}$ в один член, то получим интенсивность неотраженного света $I_{L}^{n r}$ в виде

$$
I_{L}^{n r}=\frac{4 n_{1} n_{0}}{\left(n_{0}+n_{1}\right)^{2}+n_{1}^{2} k_{1}^{2}}
$$

Для хода света из металла в диэлектрик (т. е. налево) можно закон сохранения энергии записать в виде

$$
I_{L}^{r}+I_{L}^{n r}=1
$$

Следовательно, для обоих направлений движения света через границу диэлектрик-металл закон сохранения энергии выполняется.

Если определить эквивалентную амплитуду, из которой можно вычислить интенсивность неотраженного света, то получим

$$
\mathbf{t}_{L}^{\prime}=\frac{2 n_{1}}{n_{0}+\mathbf{n}_{1}}
$$

Тот факт, что для обоих направлений движения света через границу раздела диэлектрик-металл выполняется закон сохранения энергии, дает нам основание применить принцип обратимости Стокса также и для этой границы раздела.
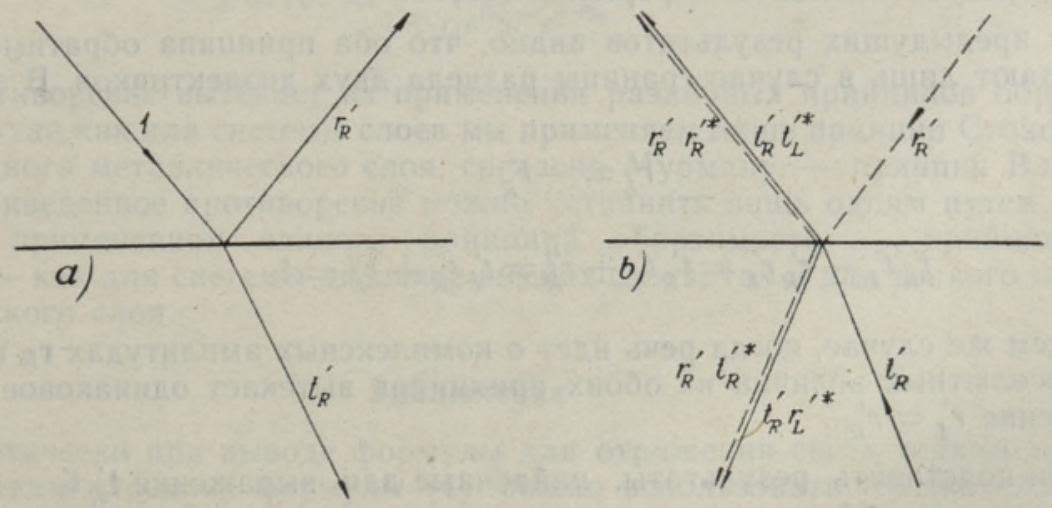

Фиг. 2. К принщипу обратимости Стокса.

Принцип обратимости Стокса требует, чтобы комплексные амплитуды изменились при обратном ходе света в амплитуды комплексно 
сопряженные; это означает, что при обратном ходе света, по Стоксу; выполняются следуюшие соотношения для напряженностей электрического и магнитного полей:

$$
\mathbf{E} \rightarrow \mathbf{E}^{*} \text { и } \mathbf{H} \rightarrow \mathbf{H}^{*} .
$$

При этом совершенно безразлично, рассматриваем ли мы простую границу раздела диэлектрик-металл или же гипотетическую границу раздела, заменяющую систему тонких слоев диэлектрика. Согласно фиг. 2 , получаем из принципа обратимости Стокса следуюшие соотношения [11]:

$$
\begin{aligned}
& \mathbf{r}_{R}^{\prime} \mathbf{t}_{R}^{\prime *}+\mathbf{t}_{R}^{\prime} \mathbf{r}_{L}^{\prime *}=0 \text { или } \mathbf{r}_{R}^{\prime *} \mathbf{t}_{R}^{\prime}+\mathbf{t}_{R}^{\prime *} \mathbf{r}_{L}^{\prime}=0 \\
& \mathbf{t}_{R}^{\prime} \mathbf{t}_{L}^{\prime *}+\mathbf{r}_{R}^{\prime} \mathbf{r}_{R}^{\prime *}=1 \text { или } \mathbf{t}_{R}^{\prime *} \mathbf{t}_{L}^{\prime}+\mathbf{r}_{R}^{\prime *} \mathbf{r}_{R}^{\prime}=1
\end{aligned}
$$

причем для абсолютных величин амплитуд одновременно выполняется закон сохранения энергии

$$
t_{R}^{\prime} t_{L}^{\prime}+r_{R}^{\prime 2}=t_{L}^{\prime} t_{R}^{\prime}+r_{L}^{\prime 2}=1
$$

Из формул (17) и (18) после несложных преобразований вытекает

$$
\mathbf{t}_{R}^{\prime} \mathbf{t}_{L}^{\prime}-\mathbf{r}_{R}^{\prime} \mathbf{r}_{L}^{\prime} \frac{\mathbf{t}_{R}^{\prime}}{\mathbf{t}_{R}^{\prime \prime}}
$$

Формулы (17) и (18) - это два соотношения, из которых можно вычислить нефренелевские комплексные амплитуды $\mathbf{r}_{L}^{\prime}$ и $\mathbf{t}_{L}^{\prime}$ :

$$
\begin{gathered}
\mathbf{r}_{L}^{\prime}=-\mathbf{r}_{R}^{\prime *} \cdot \frac{\mathbf{t}_{R}^{\prime}}{\mathbf{t}_{R}^{\prime *}} \\
\mathbf{t}_{L}^{\prime}=\frac{1-r_{R}^{\prime 2}}{\mathbf{t}_{R}^{\prime *}}
\end{gathered}
$$

Если, например, вычислить комплексную амплитуду $t_{L}^{\prime}$ из последнего соотношения, то получим для границы раздела металл-диэлектрик такой же результат, как и в формуле (16).

Из предыдущих результатов видно, что оба принципа обратимости совпадают лишь в случае границы раздела двух диэлектриков. В этом случае

$$
\begin{gathered}
r_{L}^{\prime}=-r_{R}^{\prime} \\
t_{R}^{\prime} t_{L}^{\prime}-r_{R}^{\prime} r_{L}^{\prime}=t_{R}^{\prime} t_{L}^{\prime}+r_{R}^{\prime 2}=t_{L}^{\prime} t_{R}^{\prime}+r_{L}^{\prime 2}=1
\end{gathered}
$$

В общем же случае, когда речь идет о комплексных амплитудах $\mathbf{r}_{R}$ и $\mathbf{r}_{L}$, для абсолютных величин из обоих принципов вытекает одинаковое соотношение $r_{L}^{\prime}=r_{R}^{\prime}$.

Если подставить результаты, найденные для выражения $\mathbf{t}_{R}^{\prime} \mathbf{t}_{L}^{\prime}-\mathbf{r}_{R}^{\prime} \mathbf{r}_{L}^{\prime}$ и для комплексной амплитуды $\mathbf{r}_{L}^{\prime}$ [формулы (19) и (20)] в общую формулу (1), то получим нашу пересмотренную формулу для отражения света тонким металлическим слоем 


$$
\mathrm{r}=r e^{i \bar{\partial}}=\frac{\mathrm{r}^{\prime}+\mathrm{r}^{\prime \prime} e^{i 2 \partial^{\prime}} e^{-i \mathbf{x}}}{1+\mathrm{r}^{\prime *} \mathrm{r}^{\prime \prime} e^{i 2 \partial^{\prime}} e^{-i \mathbf{x}}}
$$

(если обозначим $\frac{\mathbf{t}_{R}^{\prime}}{\mathbf{t}_{R}^{\prime \prime}}=e^{i 2 \partial^{\prime}}$ и опустим одинаковый индекс $R$ ).

\section{2. Формулы для отражения света системой тонкого слоя диэлектрика и тонкого слоя металла}

Расчеты отражения света системой тонких диэлектрических слоев и одним слоем диэлектрика не противоречат друг другу, так как при расчете отражения света одним слоем диэлектрика или же системой тонких диэлектрических слоев мы руководствуемся единым принципом обратимости, а именно - принципом Стокса. Однако в случае, если рассчитывать отражение света тонким слоем диэлектрика, соединенным с тонким металлическим слоем, мы встречаемся с определенными противоречиями. Поясним это на следующем примере.

Для отражения света тонким слоем диэлектрика, прилегающим к металлической границе раздела, выполняется соотношение

$$
\mathbf{r}_{1}=r_{1} e^{i \grave{c}_{1}}=\frac{r^{\prime}+\mathbf{r}^{\prime \prime} e^{-i x_{1}}}{1+r^{\prime} \mathbf{r}^{\prime \prime} e^{-i x_{1}}}=\frac{r^{\prime}+r^{\prime \prime} e^{-i\left(x_{1}+\alpha\right)}}{1+r^{\prime} r^{\prime \prime} e^{-i\left(x_{1}+a\right)}}
$$

Если при расчете отражения мы имеем еще и тонкий металлический слой с комплексным сдвигом фазы $\mathbf{x}_{2}$, то получим

$$
\mathbf{r}=r e^{i j}=\frac{r_{1} e^{i \hat{\sigma}_{1}}+\mathbf{r}^{\prime \prime \prime} e^{i 2 \vartheta_{1}} \cdot e^{-i \mathbf{x}_{2}}}{1+r_{1} e^{-i \hat{\partial}_{1}} \mathbf{r}^{\prime \prime \prime} e^{i 2 \partial_{1}} \cdot e^{-i \mathbf{x}_{2}}}
$$

Противоречие возникает в том случае, когда сдвиг фазы $x_{1}$ в тонком слое диэлектрика будет кратным 2л. Тогда отражение света будет таким же, как и в случае одного лишь металлического слоя без предшествующего слоя диэлектрика. В только что описанном случае мы противоречим формуле Мурмана, согласно которой при отражении света тонким металлическим слоем должно выполняться соотношение

$$
\mathbf{r}=r e^{i \delta}=\frac{r_{1} e^{i \hat{\sigma}_{1}}+\mathbf{r}^{\prime \prime \prime} e^{-i \mathbf{x}_{2}}}{1+r_{1} e^{i \hat{1}_{1}} \mathbf{r}^{\prime \prime \prime} e^{-i \mathbf{x}_{2}}}
$$

Это противоречие вытекает из применения различных принципов обратимости, так как для системы слоев мы применяем лишь принцип Стокса, а для одного металлического слоя, согласно Мурману, - принцип Власова. Приведенное противоречие можно устранить лишь одним путем, а именно, применением единого принципа обратимости - принципа Стокса - как для өистемы диэлектрических слоев, так и для тонкого металлического слоя.

\section{Заключение}

Теоретически при выводе формулы для отражения света тонким металлическим слоем из формулы (1) можно использовать соотношения, полученные либо из принципа Власова, либо из принципа Стокса. Если в общей формуле (1) использовать соотношения, вытекающие из принципа Власова, то для отражения света тонким металлическим слоем 
получаем формулу Мурмана; если же использовать соотношения, вытекающие из принципа Стокса, то получим нашу пересмотренную формулу. Обе формулы приводят к различным численным результатам, и лишь подробный анализ последних может решить, какая из формул верна. В связи с этим автору хотелось бы коротко напомнить те результаты, которые более подробно освещены в упомянутой выше книге [5]:

1. Правильной является пересмотренная формула, хотя она верна только для слабо поглощающих веществ, например полупроводников, которые больше всего подходят к предположению бесконечного множества отражений в тонком поглощающем слое. Пересмотренную формулу нельзя применить для обычных сильно поглощающих металлов, так как в таком слое не выполнено условие бесконечного множества отражений.

2. Формула Мурмана приводит приблизительно к тем же результатам, что и формула для однократного отражения света тонким металлическим слоем

$$
\mathbf{r}=r e^{i \hat{\sigma}}=\mathbf{r}^{\prime}+\mathbf{r}^{\prime \prime} e^{-i x}
$$

Поэтому формула Мурмана обеспечивает довольно хорошее совпадение с измерениями в случае сильно поглощающих металлических слоев. Но при расчете отражения света сильно поглощающим металлическим слоем можно ограничиться формулоӥ (28) для однократного отражения света тонким металлическим слоем.

3. При расчете отражения света тонким металлическим слоем необходимо помнить, что существует одна формула для однократного отражения тонким слоем, а другие формулы для двухкратного, трехкратного и т. д. до бесконечного множества отражений в тонком металлическом слое. В обоих предельных случаях, а именно - простого отражения и бесконечного множества отражений, расчеты довольно просты. В остальных случаях, т. е. при двухкратном, трехкратном и т. д. отражениях, расчеты очень усложняются. Такое различие в формулах связано с тем, что в каждом отдельном случае мы имеем разные значения комплексной амплитуды $\mathbf{r}^{\prime \prime}$. Эти значения можно определить из предельных условий (из того, что для нулевой толщины металлического слоя отражение будет таким же, как и в случае чистой подложки).

4. Вопросы отражения усложняются еще и различной структурой слоя при разных толщинах металлического покрытия, как это показал в своей микротеории Розенберг [12].

\section{Л И Т Е Р АТ У Р A}

1. П. Г. К а р д, Изв. АН Эст. ССР. Серия техн. и физ.-мат. наук, т. VII, № 4, 1958, 283.

2. V. V r b a, Csl. časopis pro fysiku, 4, 1954, 480.

3. А. В а ши ч ек, Чехосл. физ. ж., $5,1955,103$.

4. 3. Кни т тл, Чехосл. физ. ж., 8, 1958, 133.

5. A. V a šiček, Optics of Thin Films, Amsterdam, 1960.

6. А. В а ш и ч е к, Чехосл. физ. ж., 8, 1958, 135.

7. А. А. В ла сов, Макроскопическая электродинамика, М., 1955, 40.

8. F. A. Jenkins, H. E. Wh it e, Fundamentals of Optics, New York, 1950, 200.

9. 3. Книттл, Чехосл. физ. ж., 9, 1959, 133.

10. L. Dunajský. Rozbor amplitudových a energetických pomerov, Brno, 1960 (неопубликованная диссертация)

11. 3. Кни т тл, Чехосл. физ. ж., 7, 1957, 427.

12. Г. В. Р озенб е рг, Оптика тонкослойных покрытнй, М., 1958, 332. 


\title{
VALGUSE PEEGELDUMISEST ÕHUKESEL METALLIKIHIL
}

\author{
Antonín Vašiček, \\ füüsikalis-matemaatiliste teaduste doktor
}

\section{Resümee}

Valguse peegeldumise jaoks mistahes õhukesel kihil kehtib üldine valem (1). Artiklis püstitatakse küsimus, kas tänini sageli kasutatav Murmanni valem valguse peegeldumise jaoks ôhukesel metallikihil on ôige või mitte. Pööratavusprintsiibid viivad jărgmistele seostele:

a) Vlassovi printsiibist (joon. 1) tulenevad seosed (4) ja (5), mille asetamine üldisesse valemisse (1) annab Murmanni valemi tuntud kujul (6).

b) Stokes'i printsiibi põhjal (joon. 2) saadakse seosed (17) ja (18), mille järgi absoluutväärtuste jaoks kirjutatakse valem $\left(18^{\prime}\right)$. Viimane on kooskōlas energia jäävuse seadusega. Et energia jäävuse seadus on dielektriku ja metalli lahutuspinnal kehtiv mōlemas suunas, saadakse seoste (20) ja (21) asetamisel üldisesse valemisse (1) revideeritud valem (24) valguse peegeldumise jaoks ōhukesel metallikihil.

Arvutamisel tekivad raskused, kui arvutused tehakse dielektrikust-metallist kaksikkihi jaoks. Need raskused kõrvaldatakse sel teel, et rakendatakse ainuüksi Stokes'i printsiipi.

Lōpuks mainitakse lühidalt arvutustulemusi mōlema valemi - (6) ja (24) järgi. Revideeritud valem (24) kehtib ainult nörgalt absorbeerivate ainete (pooljuhtide) kohta, kus kihis toimuvate piiramata hulga peegeldumiste eeldus on ligikaudu täidetud. Harilikel tugeva absorptsiooniga metallidel on Murmanni valem (6) kooskōlas mõōtmistulemustega, sest ta vastab ligikaudu valemile (28). Tuleb siiski arvestada, et olenevalt kompleksse amplituudi $r^{\prime \prime}$ erinevatest väärtustest kehtib ühe- ja mitmekordse peegeldumise puhul metallikihis igakord eri valem, mis tegelikku arvutamist tublisti raskendab.

Olikool Brnos,

Tšehhoslovakkia Vabariik
Saabus toimetusse 4. III 1960

\section{ZUR FRAGE DER LICHTREFLEXION AN EINER DUNNEN METALLSCHICHT}

\section{Antonín Vašiček \\ Zusammenfassung}

Für die Lichtreflexion an einer dünnen Schicht gilt eine allgemeine Formel (1). Es wird die Frage gestellt, ob die bisher oft verwendete Formel von Murmann für die Lichtreflexion an einer dünnen Metallschicht richtig ist oder nicht. Prinzipien der Reversibilität führen $\mathrm{zu}$ folgenden Beziehungen:

a) das Prinzip von Vlassov (Abb. 1) zu den Beziehungen (4) und (5), die durch Einsetzen in die allgemeine Formel (1) zur Murmannschen Formel (6) führen;

b) das Prinzip von Stokes (Abb. 2) ergibt die Beziehungen (17) und (18), wonach man für absolute Werte die Formel (18') schreibt, die dem Gesetz der Erhaltung der Energie entspricht. Da dieses Gesetz für die Grenze Dielektrikum-Metall erfüllt ist, erhält man durch Einsetzen der Beziehungen (20) und (21) in die allgemeine Formel (1) eine revidierte Formel für die Lichtreflexion an einer dünnen Metallschicht (24).

Schwierigkeiten in den Berechnungen entstehen, wenn man sie für eine Doppelschicht Dielektrikum-Metall durchführt. Diese Schwierigkeiten werden dadurch beseitigt, dass das Stokessche Prinzip allein angewendet wird.

Zum Schluss werden Berechnungsergebnisse nach den beiden Formeln (6) und (24) kurz erwähnt. Die revidierte Formel (24) gilt nur für schwach absorbierende Materialien (Halbleiter), wo die Voraussetzung einer unbeschränkten Anzahl der Reflexionen in der Schicht annähernd erfüllt ist. Für gewöhnliche Metalle mit starker Absorbtion stimmt die Murmannsche Formel (6) mit den Messergebnissen überein, da sie der Formel (28) annähernd entspricht. Es muss jedoch beachtet werden, dass - bedingt durch die verschiedienen Werte der kimplexen Amplitude $r^{\prime \prime}$ - für die einmalige und mehrmalige Reflexion in der Metallschicht jeweils eine besondere Formel gilt, was die praktischen Berechnungen sehr erschwert. 